Cahiers de recherches médiévales

\title{
La conjointure de l'anomalie et du stéréotype
}

Un modèle de l'invention dans les romans arthuriens en vers

\section{Douglas Kelly}

\section{CpenEdition}

Journals

Édition électronique

URL : https://journals.openedition.org/crm/2648

DOI : $10.4000 / \mathrm{crm} .2648$

ISSN : 1955-2424

Éditeur

Honoré Champion

Édition imprimée

Date de publication : 15 décembre 2007

Pagination : 25-39

ISSN : $1272-9752$

Référence électronique

Douglas Kelly, « La conjointure de l'anomalie et du stéréotype », Cahiers de recherches médiévales [En ligne], 14 | 2007, mis en ligne le 15 décembre 2010, consulté le 15 décembre 2022. URL : http:// journals.openedition.org/crm/2648; DOI : https://doi.org/10.4000/crm.2648 


\title{
rin
}

\section{La conjointure de l'anomalie et du stéréotype : un modèle de l'invention dans les romans arthuriens en vers}

\author{
Ly roiz Artus vit la pucelle, \\ Molt le vit bien vestue et bielle. ${ }^{1}$
}

La description stéréotypée dans les romans en vers dérive évidemment de la tradition scolastique illustrée dans les arts poétiques des $\mathrm{XII}^{\mathrm{e}}$ et $\mathrm{XIII}^{\mathrm{e}}$ siècles. Les romanciers y font allusion en évoquant les clercs comme maîtres de cet art. À propos d'une belle femme par exemple, le narrateur de l'Âtre périlleux évoque ainsi l'indicible :

\author{
Ki trestout vauroit deviser \\ Et bien i vauroit aviser, \\ N'a si bon clerc dusqu'a Paris, \\ Ja tant n'i metroit son avis, \\ K'il en deïst por nule painne \\ Tout le voir en une semainne, \\ K'il n'i trespassast quoi que soit. ${ }^{2}$
}

On sait que le clerc qui passait par les bonnes écoles des $\mathrm{XII}^{\mathrm{e}}-\mathrm{XIII}{ }^{\mathrm{e}}$ siècles atteignait une maîtrise de l'art de la composition en imitant et parfois même en émulant des auteurs exemplaires de l'Antiquité comme Virgile ou Ovide et de l'époque médiévale tels que Bernard Silvestre et Jean de Hauville ${ }^{3}$. Mais écrire en français pour un public aristocratique demandait des adaptations. On les remarque notamment dans les descriptions de Chrétien de Troyes qui, dans la matière de Bretagne, s'élève comme modèle pour les auteurs des romans en vers ${ }^{4}$. Afin

\footnotetext{
${ }^{1}$ Gliglois, éd. M.-L. Chênerie, Paris, 2003, v. 155-56.

${ }^{2}$ L'Âtre périlleux, éd. B. Woledge, Paris, 1936, v. 2583-89. Cf. Girart d'Amiens, Escanor, éd. R. Trachsler, 2 t., Genève, 1994, v. 8397-402; Jehan, Les Mervelles de Rigomer, éd. W. Foerster et alii, 2 t., Dresden, 1908-15, v. 9693-96; Robert de Blois, Beaudous, éd. J. Ulrich, Berlin, 1889, v. 1508-11.

${ }^{3}$ A. N. Cizek, Imitatio et tractatio : die literarisch-rhetorische Grundlagen der Nachahmung in Antike und Mittelalter, Tübingen, 1994 ; D. Kelly, The Arts of Poetry and Prose, Turnhout, 1991, p. 57-64, 160-63 ; et Kelly, The Art of Medieval French Romance, Madison, Londres, 1992, chap. 2.

4 B. Schmolke-Hasselmann, Der arthurische Versroman von Chrestien bis Froissart, Tübingen, 1980 ; M.-L. Chênerie, Le Chevalier errant dans les romans arthuriens en vers des XII ${ }^{e}$ et XIII ${ }^{e}$ siècles, Genève, 1986 ; The Legacy of Chrétien de Troyes, éd. N. J. Lacy et alii, 2 t., Amsterdam, 1987-88 (cf. C.-A. Van Coolput, «Références, adaptations et emprunts directs », t. I, p. 333-42); L'Euvre de Chrétien de Troyes dans la littérature française: réminiscences, résurgences et réécritures, éd. Cl. Lachet, Lyon, 1997.
}

Cahiers de Recherches Médiévales, 14, 2007 
d'apprécier la survivance de la description scolastique dans les romans arthuriens en vers, il faut tenir compte de deux facteurs : le modèle de l'art que Chrétien pratique dans ses romans et l'adaptation de cet art à une langue et pour un public nouveaux.

Dans le Tournoiement Antéchrist, Huon de Mery annonce bien avant La Bruyère que « tot est dit $»^{5}$. Par exemple, quand il arrive à la fontaine périlleuse dans la forêt de Brocéliande, il la trouve «en itel maniere / Comme l'a descrit Crestiens » (v. 102-03). La fontaine et l'orage qu'Yvain déclenche, "Si con Crestiens le tesmoine", sont évoqués à nouveau dans Claris et Laris ${ }^{6}$. Mais Huon de Mery va encore plus loin. Il prétend avoir versé deux fois plus d'eau sur le perron de la fontaine, avec comme résultat un orage bien plus violent que celui que décrit Chrétien dans Yvain (Tournoiement, v. 132-48); le ciel s'ouvre littéralement de sorte qu'on voit le paradis (v. 154-61).

Or, l'auteur anonyme du Roman d'Yder critique ce genre de description qu'il appelle hyperbole. Il y a hyperbole si les romanciers «dient plus qu'il ne deivent»? En ce faisant, poursuit-il, leurs descriptions deviennent invraisemblables. Huon de Mery et l'auteur d'Yder évoquent, chacun à sa manière, les descriptions de lieux. Mais d'autres descriptions sont sujettes aux mêmes critères: comme l'affirme l'auteur d'Yder, ses remarques sont valables pour « unes descripcions / De vergiez et de paveillons / E de el», c'est-à-dire d'autre chose (Yder, v. 4446-48). Les descriptions de personnes, elles aussi, peuvent donc être hyperboliques. Le sontelles? Et si oui, pourquoi?

L'art de la description stéréotypée est enseigné dans les arts poétiques contemporains de Chrétien et de ses émules ${ }^{8}$. Pourtant selon Geoffroi de Vinsauf, la description stéréotypée est un procédé rebattu ${ }^{9}$; pour cette raison, dans sa Poetria nova, il préfère passer à d'autres motifs selon lui moins usés tels que celui du grand festin (Poetria nova, v. 622-67). Mathieu de Vendôme pour sa part recommande la description de la beauté pour les femmes, l'estimant moins digne des hommes chez

\footnotetext{
${ }^{5}$ Huon de Mery, Li Tornoiemenz Antecrit, éd. G. Wimmer, Marburg, 1888, v. 8.

${ }^{6}$ Claris et Laris, éd. J. Alton, Tübingen, 1884, Amsterdam, 1966, v. 627.

${ }^{7}$ Yder, éd. A. Adams, Cambridge, 1983, v. 4449. Voir E. Faral, Les Arts poétiques du XII et XIII $e^{e}$ siècle, Paris, 1924, p. 84 ; Ph. Ménard, Le Rire et le sourire dans le roman courtois en France au moyen âge (1150-1250), Genève, 1969, p.638-49; D. A. Monson, "La «surenchère »chez Chrétien de Troyes", Poétique, 70, 1987, p. 231-46. Le romancier anonyme d'Yder pensait peut-être aussi aux romans d'antiquité où l'hyperbole est plus importante que dans la matière de Bretagne. Cf. A. Petit, Naissances du roman: les techniques littéraires dans les romans antiques du XII $e^{e}$ siècle, 2 t., Paris, Genève, 1985, p. 540-41: «un goût certain pour l'insolite... se manifeste dans tous nos romans antiques» . Voir aussi U. Schöning, Thebenroman-Eneasroman-Trojaroman : Studien zur Rezeption der Antike in der französischen Literatur des 12. Jahrhunderts, Tübingen, 1991, p. 232-47; C. Croizy-Naquet, Thèbes, Troie et Carthage : poétique de la ville dans le roman antique au XII siècle, Paris, 1994, chap. II.

${ }^{8}$ Faral, op.cit. p. 75-84 ; Cizek, op.cit., p. 134-36 ; Kelly, op.cit. 1991 [n. 3], p. 71-78. Cf. J.Y. Tilliette, «La Descriptio Helenae dans la poésie latine du $\mathrm{XII}^{\mathrm{e}}$ siècle », Bien dire et bien aprandre, 11, 1993, p. 419-32.

${ }^{9}$ Trita / Et vetus (Geoffroi de Vinsauf, Poetria nova, v. 622-23, dans Faral, op. cit., p. 216); cf. son Documentum, II.2.4 (Faral, op. cit., p. 272).
} 
qui d'autres qualités sont plus importantes que la parfaite beauté ${ }^{10}$. Girart d'Amiens paraît se conformer à cette règle en déployant une ample description traditionnelle de la femme et amie d'Escanor le Beau (Escanor, v. 8407-64), tandis que celle de la beauté d'Escanor lui-même est plus brève (v. 8465-76).

Ailleurs Mathieu enseigne qu'une description doit faire valoir ce qui est typique du personnage qu'on décrit. Un roi, par exemple, ne doit pas avoir les attributs d'un pape (Ars, $\S 1: 65-66)$; on exclut aussi dans les romans jusqu'aux rides et verrues sur la peau des belles femmes. ${ }^{11}$

Sans doute la prolifération des descriptions de la beauté merveilleuse dans les romans en vers peut nous faire penser que nos romanciers disent plus qu'ils ne doivent. Pourtant ils continuent à les introduire dans leurs œuvres ${ }^{12}$. Les descriptions physiques de belles dames y abondent; celles de beaux hommes n'y manquent pas non plus ${ }^{13}$. Chrétien pour sa part donne un exemple élaboré de la description d'une

\footnotetext{
${ }^{10}$ Mathieu de Vendôme, Ars versificatoria, éd. F. Munari, Rome, 1988, §1 :67-68. Cf. An Early Commentary on the "Poetria nova " of Geoffrey of Vinsauf, éd. M. C. Woods, New York, Londres, 1985, p. 58 : docet quomodo prolongari possit materia... si aliqua res secundum suam proprietatem diligenter describatur.

${ }^{11}$ Le Chevalier as deus espees, éd. P. V. Rockwell, à paraître chez D. S. Brewer, Cambridge, 2006, v. 4292 (je remercie le professeur Rockwell d'avoir bien voulu me communiquer le texte de son édition avant la publication).

${ }^{12}$ Selon la Rhetorica ad Herennium, de telles descriptions sont plus mémorables, notamment si elles contiennent des éléments inattendus ou des anomalies; voir J. Enders, "Memory, Allegory, and the Romance of Rhetoric », Yale French Studies, 95, 1999, p. 53.

${ }^{13}$ Sur la description chez Chrétien, voir Kelly, «The Art of Description », Legacy, t. I, p. 197207 ; C. Blons-Pierre, «L'esthétique de la description des personnages chez Chrétien de Troyes ", Bien dire et bien aprandre, 11, 1993, p. 55-68. A. M. Colby, The Portrait in Twelfth-Century French Literature: An Example of the Stylistic Originality of Chrétien de Troyes, Genève, 1965, p. 14-22, offre de nombreux exemples du XII ${ }^{\mathrm{e}}$ siècle. Y ajouter, pour les femmes: Raoul de Houdenc, Meraugis de Portlesguez, éd. M. Szkilnik, Paris, 2004, v. 232-42 ; Renaut de Bâgé, Le Bel Inconnu, éd. K. Fresco, New York, 1992, v. 1525-54, 1931-43, 2217-58, 2395-414, 2425-31, 3261-300, 3949-94, 4344-55 ; Raoul de Houdenc, La Vengeance Raguidel, éd. G. Roussineau, Genève, 2004, v. 5161-67, 5769-93; Durmart le Galois, éd. J. Gildea, 2 t., Villanova (PA), 1965-66, v. 159-64, 1889-939 ; Âtre, v. 21-31, 2575-89, 4076-89 (une anomalie où "Biautés... a orguel s'acoste »); Guillaume le Clerc, Fergus, éd. W. Frescoln, Philadelphie, 1982, v. 1519-89; Yder, v. 6470-78; Floriant et Florete, éd. A. Combes et R. Trachsler, Paris, 2003, v. 2884-921 ; Gliglois, v. 134-45 ; Escanor, v. 3119-40. Et pour les hommes : Raguidel, v. 2084-93 ; Âtre, v. 86-89, 1558-603, 3590-650 ; Fergus, v. 1240-52, 1634-45 ; Yder, v. 275-79, 1144-74, 5923-26; Floriant, v. 739-67, 817-41 ; Chevalier as deus espees, v. 1510-16, 1528-38, 1555-66, 1586-91, 265997 ; Gliglois, v. 1-22 ; Escanor, v. 435-54. Voir aussi M. Klose, Der Roman von Claris und Laris in seinen Beziehungen zur altfranzösischen Artusepik des XII. und XII. Jahrhunderts unter besonderer Berücksichtigung der Werke Crestiens von Troyes, Halle/S., 1916, p. 41-42; M.-M. Castellani, «D'un portrait l'autre, le renouveau de l'écriture descriptive dans Athis et Prophilias », Par les mots et les textes: mélanges de langue, de littérature et d'histoire des sciences médiévales offerts à Claude Thomasset, ed. D. James-Raoul et O. Soutet, Paris, 2005, p. 135-42.
} 
femme (plus de 80 vers) dans Philomena ${ }^{14}$, avant d'y revenir plus brièvement (40 vers) dans son premier roman arthurien ${ }^{15}$. Là, dit-il, Nature façonna en Enide un chef d'œuvre à la fois unique (Erec, v. 418-21) et virtuellement archétype. "Que diroie de sa beauté ?» se demande Chrétien, quand Dieu lui-même n'aurait su faire mieux (v. 435-37). Il clôt donc le catalogue des perfections d'Enide au bord de sa robe. Que dire en effet si tant d'autres ont déjà détaillé à l'envi les attributs de cette beauté, splendide et peu variable, y compris Chrétien lui-même dans Philomena? Où est donc la limite entre la description à faire et celle qui dit plus qu'il ne faut, devenant par conséquent hyperbolique dans le sens péjoratif du roman d'Yder?

L'art poétique veut que les attributs de la personne soient décrits convenablement et en conformité avec un modèle idéal. On remarque aussi que la description des vêtements peut être complétée par celle du cheval du chevalier ou bien du beau château où demeure le chevalier ou la dame. Le château et les terres du domaine sont fréquemment à l'origine du surnom qui révèle la qualité de la personne, selon la mère de Perceval ${ }^{16}$. Souvent on ne connaît le personnage que par ce seul surnom, comme c'est le cas pour la reine des Traverses et de la reine de la Blanche Montagne dans Escanor. Ces noms signalent la haute noblesse et aussi, dans ce roman, les domaines que ces deux reines apportent comme dot à ceux qu'elles épousent (v. 5060-64, 13700-05). Les terres des dames sont à l'origine de maintes aventures dans les romans arthuriens, depuis l'épisode du comte Allier dans Yvain jusqu'à Dionise dans Rigomer (v. 102-09, 6151-54). Dans Meraugis, on signale le fait que Lidoine occupe ses terres sans contestation (v. 116-24).

Ailleurs, les fêtes et les processions peuvent servir à exalter celui qui les organise. Arthur est remarquable de ce point de vue. De tels paremenz qui ornent la personne incluent les vêtements et les demeures. Dans l'Escanor Girart d'Amiens décrit le cortège qui accompagne Escanor le Beau vers un combat judiciaire avec Gauvain. Comme les vêtements ornent («paremenz», v. 8106) les bacheliers et les demoiselles dans le cortège, la noblesse de cette jeunesse joyeuse et belle est «paremens»d'Escanor et de sa dame (v. 8086). Ils font partie de leurs nobles attributs (v. 19338), c'est-à-dire « de quanqu'afiert a preudome» (v. 8202). Ceci fait contraste, ailleurs dans le même roman, avec l'armure laide et diabolique de Galentinet (v. 7866-911) qui essaie d'assassiner Escanor le Beau et avec l'abattement et l'angoisse de Gauvain qu'on accuse de cette félonie, «contenance » qu'Arthur lui reproche comme indigne d'un chevalier (v. 8298-300).

Mais les romanciers ne suivent pas toujours les consignes des arts poétiques de leur époque. Par exemple, les festins, scènes nouvelles que recommande Geoffroi de Vinsauf, sont plutôt négligés ou abruptement terminés :

${ }^{14}$ Vv. 124-207 (p. 166-74) dans Chrétien de Troyes, Pyrame et Thisbé, Narcisse, Philomena, éd. E. Baumgartner, Paris, 2000.

${ }^{15}$ Chrétien de Troyes, Erec et Enide, éd. J.-M. Fritz, Paris, 1992, v. 402-42.

${ }^{16}$ Chrétien de Troyes, Perceval ou le Conte du graal, éd. K. Busby, Tübingen, 1993, v. $557-$ 62. Les variantes remplacent «sornon» par «non» (see p. 430, n. 561-562). Cf. «par le non connoist on la gent» (Le Roman de Tristan en prose, éd. Ph. Ménard, t. I, Genève, 1987, $\S 78$ : 19-20). Voir sur le nom et l'invention de l'intrigue R. Trachsler, Disjointuresconjointures : étude sur l'interférence des matières narratives dans la littérature française du Moyen Age, Tübingen, Bâle, 2000, p. 9-47. 
Des més ne vous quier fere conte,

Mes onques rois ne duc ne conte

Ne furent mieux assis a table :

Ne vous en quier fere autre fable. (Floriant, v. 125-28) ${ }^{17}$

On trouve même l'abréviation d'un combat chez Chrétien. «La bataille fu fors et dure. / De plus deviser n'en ai cure, / Que paine gastee me samble» (Perceval, v. 3927-29) ${ }^{18}$. Mais cette abréviation n'a pas fait école. On dirait que, dans Perceval, Chrétien s'allie à Erec en préférant décrire l'amour au combat. Mais là aussi il y a des coupures. Par exemple, dans la Charrette, Chrétien supprime la dernière étape d'un gradus amoris : «Mes toz jorz iert par moi teüe, / Qu'an conte ne doit estre dite $»^{19}$. Moins précis, il constate seulement

Que il lor avint sanz mantir

Une joie et une mervoille

Tel c'onques ancor sa paroille

Ne fu oïe ne seüe. (v. 4676-79) fabliaux.

L'allusion remplace le détail cru de la fin du Roman de la rose et des

En ce qui concerne les descriptions plus abstraites des attributs caractéristiques du chevalier dans les romans arthuriens, on constate une tendance à les répéter brièvement à des moments convenables, par exemple, lors d'un exploit chevaleresque remarquable ou bien d'une action particulièrement courtoise, afin de relever la valeur exemplaire du héros. Lorsque Escanor le Beau se défend contre l'armée du roi Arthur: «La fu moustree la hautece / Du Bel Escanor ou Prouece / Avoit pris repaire et ostage»(Escanor, v. 19697-99). Ainsi évoque-t-on la vertu conventionnelle du chevalier manifestée dans chaque aventure épisodique.

On découvre donc une tendance à abréger, comme dans le portrait d'Enide, que sa robe cache, empêchant ainsi la description physique intégrale, tout en permettant au narrateur d'entamer une description vestimentaire, ou bien une description morale, comme c'est le cas pour Lidoine dans Meraugis de Portlesguez.

Dans ce roman, Meraugis estime chez Lidoine la beauté morale autant que la beauté physique (Meraugis, v. 10-87). Il reconnaît également une hiérarchie de ces

\footnotetext{
${ }^{17}$ De pareilles abréviations se trouvent, par exemple, dans Claris, v. 373-76 (une fête), 978-81 (deux lits), 1278-81, 5011-18 (une ville), 12419-20 (un repas); Rigomer, v. 9267-68 et 12722-23 (repas), 13331-32 (un tournoi), 15909-10 (un mariage) ; Jean Froissart, Méliador, éd. A. Longnon, 3 t., Paris, 1895-99, v. 11048-49 (un repas).

${ }^{18}$ Les scribes n'étaient pas toujours d'accord avec Chrétien le narrateur; voir Perceval, appendices 1-3 dans l'éd. Busby. Rigomer abrège souvent les combats (v. 8534-38, 8836-40, 9393-98 ; voir aussi Claris, v. 14583, 24498-507), mais pas toujours; on trouve aussi une longue description d'une partie d'un combat dont la fin est abrégée (v. 11203-486 et 1148790).

${ }^{19}$ Chrétien de Troyes, Le Chevalier de la charrette ou le Roman de Lancelot, éd. Ch. Méla, Paris, 1992, v. 4680-81.
} 
valeurs dans laquelle la beauté morale prime la beauté du corps ${ }^{20}$. Lidoine n'est pas laide ; c'est pourquoi Gorvain Cadruz l'aime, et c'est selon lui la raison suffisante de son amour, comme Lidoine qui ne peut aimer Bergis le Louche parce que Dieu l'a fait si laid (v. 3722-37, 3831-45). Meraugis prétend pour sa part qu'il aimerait une dame courtoise même si elle était laide (v. 571-83). Intempérance ou exagération qui frise l'hyperbole? Toujours est-il qu'on trouve dans les romans quelques laides bien aimées, par exemple Rose Espanie aimée de Girflet dans le Bel Inconnu (v. 172735). Mais c'est une anomalie ${ }^{21}$. Si l'auteur du Bel Inconnu concède à Rose Espanie une belle robe et un chevalier amoureux, il ne semble pas reconnaître chez elle ${ }^{22}$ les qualités morales qui auraient pu justifier la préférence de Girflet si celui-ci partageait les convictions de Meraugis. L'amour rend Girflet aveugle. Son amour pour Rose Espanie ne peut nous aider à apprécier l'opinion de Meraugis qui, d'ailleurs, ne risque rien car la beauté stéréotypée de Lidoine est corporelle et morale.

Il faut signaler aussi des cas surprenants de personnages ambigus qui ont, comme Bergis le Louche, des traits bien plus anormaux que les vêtements provisoires d'Enide. On les trouve dans la description du corps, des vetements et du caractère aussi bien que de l'âge, de l'état dans la société aristocratique du moyen âge et $d u$ sexe - topoi de la description scolastique ${ }^{23}$. On s'étonne qu'un jeune chevalier comme Gliglois soit préféré à Gauvain comme amant, sans la tonalité de farce et de misogynie qui accompagne de tels motifs dans Raguidel et Le Chevalier à l'épée $e^{24}$. Encore plus inattendu peut-être est le cas du chevalier excellent qui, malgré ses défauts physiques, parvient à gagner la dame; par exemple, dans Raguidel, Ydain choisit Druidan. Pourquoi préfère-t-elle à Gauvain ce chevalier bossu aux membres parfaits mais si mal joints et disposés (v. 4163-211) qu'il en est laid? «Por ce ai non Druÿdain / que je doi estre drus Ydain, / ele ma drue et je ses drus» (v. 4349-51) et parce qu'un oracle a prédit cette union incongrue (v. 4356$60)^{25}$. Evidemment Ydain n'a pas les mêmes goûts qu'Hermondine dans Meliador quand elle refuse Camel de Camois en partie parce qu'il est somnambule (v. 632455). Dans le roman occitan Jaufre, on conteste la noblesse et la chevalerie de Fellon d'Albarra à cause de sa profonde méchanceté. Ce défaut moral se manifeste dans des

${ }^{20}$ Cf. Yder, v. 6506-12 ; Tornoiemenz, v. 1994-99.

${ }^{21}$ Dans la Seconde Continuation du Perceval, une autre laide, Rosete amie du Beau Mauvais, est peut-être une fée qui se transforme plus tard en beauté splendide (The Continuations of the Old French "Perceval», t. 4, éd. W. Roach, Philadelphie, 1971, v. 23170-200, 23526-32). Une métamorphose semblable dans le Bel Inconnu transforme Blonde Esmeree de guivre en beauté stéréotypée.

${ }^{22}$ Mais la description est incomplète à cause d'une lacune dans le manuscrit.

${ }^{23}$ Voir Kelly, «Les fées et les arts dans la représentation du Chevalier de la charrette », dans Lancelot, éd. D. Buschinger, Göppingen, 1984, p. 85-97.

${ }^{24}$ Ménard, op.cit., p. 222-23, 232-33.

${ }^{25}$ Voir aussi Raguidel, éd. Roussineau, p. 390, note au v. 4356. Le même problème se manifeste dans Sone de Nansay quand Henri, frère de Sone et roi, mais «Povrement... enfiguré » (Sone de Nausay, éd. M. Goldschmidt, Tübingen, 1899, v. 72), doit trouver une épouse (v. 15446-56, 15498-502). Sur le titre de ce roman, voir Cl. Lachet, Sone de Nansay et le roman d'aventures en vers au XIII' siècle, Paris, Genève, 1992. 
traits bestiaux dans sa description : tête plus grande que celle d'un bœuf, chaque œil plus gros qu'œuf, bouche plus grande que celle d'un léopard... (v. 8764-83) ${ }^{26}$, traits d'habitude réservés aux vilains tels que le Gardien de troupeaux dans Yvain (v. 286311) et les gardiens des terres de la fée Madone dans Claris et Laris (v. 8369-84). Les traits du vilain, cible comique sans doute ${ }^{27}$, ne sont pas pour plaire quand on les retrouve chez un grand chevalier. La laideur physique trahit ainsi la bassesse morale comme la beauté révèle la bonté. Fellon d'Albarra se rétablit pourtant. Comme souvent dans les romans arthuriens, le chevalier vicieux, en se constituant prisonnier à la cour d'Arthur, recouvre une forme de virginité - sa noblesse innée - et se transforme immédiatement en chevalier digne de la Table Ronde. Le défaut moral s'efface pour ne laisser que la noblesse congénitale.

D'où vient cet engouement pour ce genre de description stéréotypée, engouement qu'on retrouvera dans la littérature courtoise jusque dans le $X^{\mathrm{e}}$ siècle $?^{28}$ Selon un autre maître dans l'enseignement de l'art poétique, un roi doit être décrit comme l'appui de ses chevaliers et comme modèle de courage, mais pas comme blandus amator uxoris, c'est-à-dire, comme suave amant de son épouse ${ }^{29}$. Or, cette anomalie se retrouve dans les romans. Erec, d'abord prince et ensuite roi, n'est-il pas blandus amator uxoris ? Si ce trait est une anomalie dans la description stéréotypée du chevalier arthurien, il constitue aussi une adaptation originale d'un phénomène banal dans la tradition médiolatine à laquelle Chrétien a emprunté son art de la description. Mais avant d'examiner de plus près l'anomalie d'Erec, il nous faut compléter nos remarques sur la description stéréotypée à l'époque de Chrétien et après lui.

On sait que la description intégrale de la beauté comporte trois éléments : le corps, les vêtements et le caractère moral et social ${ }^{30}$. La beauté physique est comme l'émanation de la vertu intérieure, comme chez Andrievete, l'amie de Keu dans Escanor, qui, selon Arthur, est «bele et bone et loial» car elle est fille de roi, «Et samble une sadete ymage / De la biauté qui est en li» (v. 23432-35). Les portraits que j'ai mentionnés de Lidoine et d'Enide sont des stéréotypes, mais ils s'écartent l'un de l'autre dans la description de leurs vêtements. Pour Lidoine, Raoul est bref: elle serait la plus belle même si elle portait une robe trouée aux coudes (v. 150-51) ${ }^{31}$, ce qui rappelle bien sûr Enide. Mais chez celle-ci, l'anomalie vestimentaire précède l'évocation de la beauté. Les vêtements malséants de la jeune femme provoquent l'étonnement d'Erec. C'est qu'ils font contraste avec la noble beauté que la robe couvre en partie. Si la beauté d'Enide est quasi divine, la robe trouée et usée aux

\footnotetext{
${ }^{26}$ Voir Chênerie, op.cit., p. 270.

${ }^{27}$ Ménard, op.cit., p. 168-73.

${ }^{28}$ Chez Christine de Pizan, par exemple, dans le Dit de Poissy, v. 1477-1608, The Love Debate Poems, éd. B. K. Altmann, Gainesville (FL), 1998, p. 203-74, et la Belle Heaumière de Villon.

${ }^{29}$ Milicie baculus rex est et blandus amator / Vxoris ; fortes precipit esse suos (Jean de Garlande, Parisiana poetria, éd. T. Lawler, New Haven (CT), 1974, p. 86 :75-76).

${ }^{30}$ Faral, op. cit., p. 79-81.

${ }^{31}$ Ailleurs Raoul évoque les belles robes des dames qui sont juges du débat entre Meraugis et Gorvain (Meraugis, v. 878).
} 
coudes $^{32}$ est vieille et ignoble. Erec s'informe de l'anomalie créée par le contraste entre la belle Enide et ses vêtements pauvres : "De tant povre robe et si vil / Por qu'est vostre fille atornee, / Qui tant par est bele et sennee?» (Erec, v. 506-08) La réponse du père - les guerres l'ont ruiné ${ }^{33}$ - explique l'anomalie, sans enlever le scrupule : la belle Enide doit être mieux vêtue.

Son père aurait pu la faire habiller convenablement. L'oncle d'Enide, qui est comte et que les guerres n'ont évidemment pas appauvri, veut bien habiller sa nièce comme il faut. Mais le père d'Enide attend mieux, en l'occurrence un prince digne de la beauté de sa fille, un prince qui n'est ni pauvre ni vilain, et qui saura vêtir majestueusement sa beauté merveilleuse. Et voilà Erec, prince très riche et très beau $^{34}$. Erec est bien renommé et plus encore aimé. Parmi les chevaliers de la Table Ronde, seul Gauvain le devance (v. 1686-89). Si sa beauté n'est pas divine, il est beau d'une beauté parée de prouesse et de gentillesse. Fils de roi, il n'a pas encore vingt-cinq ans. Le vasselage orne cette beauté. "Que diroie de ses bontez?» (v. 93), se demande le narrateur, aussi abruptement qu'il a terminé la description d'Enide : "Que diroie de sa beauté ?»Ces questions, tout en éludant habilement l'hyperbole condamnée dans le Roman d'Yder, rappelle pourtant le stéréotype que chacun incarne à sa manière ${ }^{35}$.

Mais il ne faut pas oublier que le futur époux d'Enide n'est pas habillé convenablement non plus. Lorsqu'il paraît comme escorte de la reine, Erec «N'ot arme o lui aportee / Fors que tant solement s'espee» (v. 103-04). Ainsi désarmé, ce chevalier parangon de vasselage ne peut venger immédiatement ni la «meschine» fille de roi qui accompagne la reine lorsque le nain du chevalier Yder la fouette, ni la reine elle-même offensée par cet acte. Erec quitte la reine pour suivre Yder avec l'espoir de trouver l'armure qui lui fait défaut. Le père d'Enide la lui fournit comme il lui donnera ensuite sa fille en mariage. Une fois l'affront vengé et la beauté suprême d'Enide démontrée dans le combat pour l'épervier, Erec se conforme au modèle de son futur beau-père en refusant la proposition de l'oncle d'Enide d'habiller sa nièce quand elle doit se rendre à la cour du roi Arthur. Une fois là, Enide obtient des vêtements de reine, vêtements qui lui conviennent parce qu'elle deviendra princesse en épousant Erec, princesse et, lors du dénouement du roman, reine couronnée à Nantes devant la même cour. C'est alors qu'on peut dire que la description stéréotypée d'Enide est achevée. Cette description est en fait intégrée dans l'intrigue romanesque qui, si je puis dire, vêt et orne les personnages au fur et à mesure qu'elle avance.

\footnotetext{
${ }^{32}$ Sur les divergences dans les manuscrits, voir R. Middleton, «Enide's See-through Dress », Arthurian Studies in Honour of P. J. C. Field, éd. B. Wheeler, Cambridge, 2004, p. 143-63.

${ }_{33} \mathrm{Au}$ contraire, Perceval, étant encore nice, n'a aucune réaction quand il voit la belle Blanchefleur pour la première fois; voir Blons-Pierre, op.cit., p. 58-60. Blanchefleur est habillée convenablement bien que les guerres l'aient privée de quoi nourrir convenablement ses hommes.

${ }^{34}$ M.-M. Castellani, «La description du héros masculin dans Erec et Enide de Chrétien de Troyes », Bien dire et bien aprandre, 11, 1993, p. 105-17.

${ }^{35} \mathrm{Cf}$. Fr. Chambefort, «Le topos de l'indescriptible dans les portraits romanesques au XII siècle », Bien dire et bien aprandre, 11, 1993, p. 119-29.
} 
Plutôt que d'élaborer, comme pour les descriptions de la beauté dans la tradition médiolatine, Chrétien choisit deux formes d'adaptation. Soit qu'il coupe court, notamment lorsqu'il arrête la description du corps d'Enide là où sa robe le couvre, soit qu'il rappelle brièvement à divers moments les qualités caractéristiques d'un personnage, comme dans cet exemple bref: "Mout fu li chevaliers curtois. / Quant il vit Lanselot armé, / Cortoisement l'a salüé » (Rigomer, v. 1398-1400). Semblablement, Chrétien rappelle le vasselage d'Erec après les v. 91-92 - «Onques nuns hom de son aage / Ne fu de greignor vasselage ${ }^{36}{ }$ - lors de sa décision de suivre Yder (v. 231), et quand il décrit la libération de Cadoc des mains des géants. Enide elle-même décline les vertus de son mari dans la Joie de la cour : "N'est or pas a esprover / Ne d'onor ne de vasselage»(v. 6298-99). On constate la même tendance pour d'autres attributs tels que pröece, bonté et cortoisie ${ }^{37}$.

Nous avons mis en évidence les anomalies vestimentaires dans les descriptions stéréotypées et d'Erec et d'Enide. Chez Enide, c'est une robe malséante qui surprend. Chez le jeune chevalier, ce sont les armes et l'armure qui manquent. L'anomalie se manifeste également dans les actes, surtout chez Erec. On peut même dire qu'Erec reste un chevalier aberrant d'un bout à l'autre de son roman. Il ne prend pas part à la chasse au blanc cerf, préférant accompagner la reine tandis que les autres chevaliers de la Table Ronde se lancent avec le roi dans la forêt. Plus tard il ne va plus aux tournois, préférant-blandus amator uxoris - rester auprès de la princesse Enide. C'est un chevalier qui ne chasse ni ne tournoie.

Le tournoi, comme la chasse, est une activité chevaleresque coutumière, voire stéréotypée. Les hommes d'Erec se plaignent de l'absence de cet amant fervent de son épouse. Où sont en effet la prouesse et le vasselage de ce prince tant vanté comme un des meilleurs chevaliers de la Table Ronde? Enide est informée de leurs murmures avec les conséquences qu'on sait. Mais, on ne se le rappelle pas assez souvent, après son mariage Erec ne participe plus à aucun tournoi dans ce roman $^{38}$. Cette ellipse ${ }^{39}$ ne sera comblée que bien plus tard, dans l'adaptation bourguignonne du roman de Chrétien, où, après la quête, Erec organise un grand tournoi et y triomphe $e^{40}$.

\footnotetext{
${ }^{36}$ "Barnage » (v. 4487) dans l'édition citée ; "vaselage » se trouve parmi les variantes.

${ }^{37}$ Voir M.-L. Ollier, Lexique et concordance de Chrétien de Troyes d'après la copie Guiot, $2^{\mathrm{e}}$ éd., Montréal, Paris, 1989.

${ }^{38}$ Kelly, «Le sens de la quête dans l'Erec et Enide de Chrétien de Troyes «, Romania, 92, 1971, p. 345-46, 351-53 ; cf. S. Kay, Courtly Contradictions : The Emergence of the Literary Object in the Twelfth Century, Stanford (CA), 2001, p. 330 n. 30.

${ }^{39}$ Terme emprunté à M. Gosman, «'L'Historia malmenée' : l'idéalisation du pouvoir dans les romans antiques ", Bien dire et bien aprandre, 10, 1992, 57.

${ }^{40} L^{\prime}$ «Histoire d'Erec» en prose, éd. M. Colombo Timelli, Genève, 2000, p. 209-11. Hartmann von Aue ne fait pour sa part qu'une allusion ambiguë à une fête («hôchzît») lors du retour d'Erec et Enide dans le royaume hérité de son père (Erec, éd. A. Leitzmann, $4^{\mathrm{e}}$ éd. L. Wolff, Tübingen, 1967, v. 10056) : «man mohte eht dâ grôze kraft / von rittern und von vrouwen / wol sehs wochen shouwen» (v. 10077-79). Pendant ces six semaines, on a pu monter un ou plusieurs tournois, mais Hartmann ne le dit pas. Chrétien ne dit rien du retour éventuel d'Erec dans son royaume à Carnant; chez Hartmann, c'est là plutôt qu'à Nantes qu'il est couronné (v. 10001, 10064-65).
} 
La conjointure du stéréotype et de l'anomalie se retrouve dans les autres romans de Chrétien. Elle constitue donc un modèle de la description pour ses émules. Si, par exemple, Lancelot du Lac est le meilleur chevalier après Gauvain et Erec (Erec, v. 1690), dans la Charrette il est l'amant de la reine. C'est une distinction, certes, mais ne le rend-elle pas coupable de lèse-majesté et du péché d'adultère ? Pourtant, cet amour exceptionnel promeut Lancelot au rang de meilleur chevalier du monde. Et Perceval? Je n'ai qu'à nommer ce jeune nice ${ }^{41}$ pour que revienne à la mémoire la description singulière du jeune homme inapte, parfois lourdaud et mal vêtu; pourtant, il est destiné à être le meilleur chevalier du monde selon la belle demoiselle qui rit (v. 1039-44). L'anomalie est marquée comme chez Enide par le contraste entre beauté physique et vêtements malséants.

Et les femmes? La beauté stéréotypée de Soredamors, celle qui "desdaigneuse estoit d'amors $»^{42}$ est longuement décrite dans Cligés ${ }^{43}$. Pourtant, elle

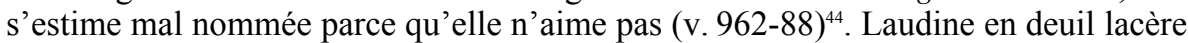
littéralement sa beauté stéréotypée, ce qui provoque la pitié mais aussi l'amour d'Yvain, qui n'a d'yeux que pour cette beautét ${ }^{4}$. Mais leur mariage n'empêchera pas le nouvel époux d'oublier son épouse dans les tournois qu'Erec néglige pour rester auprès d'Enide.

A-t-on suffisamment remarqué ces anomalies dans les descriptions stéréotypées chez Chrétien? Les a-t-on imitées? Il convient maintenant, dans l'espace dont je dispose, d'examiner plus en détail quelques exemples de la conjointure de l'anomalie et du stéréotype parmi les romans arthuriens écrits après Chrétien.

Le modèle de l'anomalie dans la description se retrouve dans le Bel Inconnu. J'ai déjà fait allusion à Girflet, amant de la très laide Rose Espanie. Celle que Guinglain aime, la Pucelle aux blanches mains, est séductrice et chaste à la fois dans la scène où, contrairement à Blanchefleur dans le Conte du graal, elle quitte brusquement le lit de l'Inconnu pour éviter la luxure (v. 2451) à laquelle elle s'adonne sans scrupule plus tard. Guinglain lui-même est parfait, lui dit-elle ailleurs, «fors tant que ne savés amer» (v. 4430). Le chevalier semble lui donner raison en la quittant pour épouser Blonde Esmeree. Chacune de ces femmes a droit à plusieurs descriptions stéréotypées (voir la n. 13). Mais le rapport entre elles rend perplexe, car c'est la Pucelle aux blanches mains qui inspire la quête de Guinglain pour libérer Blonde Esmeree de la «prison» dans laquelle sa beauté est enfermée. C'est à ce serpent tellement laid que Guinglain doit donner le "fier baisier» qui permet à Blonde Esmeree, telle le papillon qui émerge de la chenille, de retrouver sa beauté.

Le stéréotype correspond à ce qui est estimé comme à la fois idéal et normal parce que conforme à une norme préconçue. L'anomalie provoque donc la

\footnotetext{
${ }^{41}$ Sur les anomalies dans la conduite de Meraugis, d'abord un nice, puis un sage, voir M. Szkilnik, éd., Meraugis, p. 14-21.

${ }^{42}$ Chrétien de Troyes, Cligés, éd. S. Gregory et C. Luttrell, Cambridge, 1993, v. 446.

${ }^{43}$ Blons-Pierre, op.cit., p. 57-58.

${ }^{44}$ Voir S. Bianchini, «Interpretatio nominis e pronominatio nel Cligès di Chrétien de Troyes », Vox Romanica, 61, 2002, p. 181-221.

${ }^{45}$ C. Álvares et A. Diogo, "Laudine : entre fin Amors et Misogyne», Bien dire et bien aprandre, 11, 1993, p. 17-19.
} 
consternation parce qu'elle semble être une anormalité et, par conséquent, un défaut. Le contraste peut rappeler les jeux-partis. Par exemple, au début de l'Âtre périlleux, Gauvain promet de défendre une pucelle qui arrive à la cour. Quand un chevalier enlève celle-ci, Gauvain se trouve devant un dilemme : doit-il aller immédiatement à son secours, ou bien attendre la fin du repas par politesse (v. 210-15). L'indécision le fait s'attarder, inaction fustigée comme «desconvenue» par Keu (v. 234-41). On en trouve peut-être un modèle dans l'hésitation de la cour au début de la Charrette lors de l'enlèvement de Guenièvre. Là aussi Gauvain réagit après avoir hésité.

Le cas de Perceval rappelle certainement à la mémoire l'emprunt - voire le plagiat - de la description de Blancheflor pour Galiene dans Fergus et pour Clarie dans Cristal et Clarie $^{46}$. Dans le roman de Guillaume le clerc, Galiene tombe amoureuse du jeune Fergus, chevalier non seulement inapte, mais aussi-encore une anomalie - fils d'une mère noble et d'un père riche mais de naissance roturière ${ }^{47}$. Pourtant, comme chez Perceval, des personnes perspicaces remarquent que Fergus n'est pas vilain. Semblablement à la belle Enide, dont les vêtements font contraste avec la beauté naturelle, et au beau fils nommé Perceval le Galois aux qualités apparemment innées, la beauté de Fergus fait contraste avec ses vêtements grossiers et ses maladresses initiales ${ }^{48}$. Le début de Fergus introduit Perceval, ce qui sert à rappeler ce personnage et à le comparer à Fergus. Pourtant, la mère de Fergus constate que son fils «est biaus, bien sanble vasal» (Fergus, v. 509), et donc que « Encor puet a grant pris venir» (v. 512) $)^{49}$. Pour sa part, Arthur «Bien a noté a son vïaire / Que il estoit de bonne gent»(v. 882-83). Comme ailleurs, la beauté annonce la noblesse. Ce qui changera ne sera pas cette beauté, mais, comme chez Enide et chez Perceval, l'élément plus superficiel de sa personne, les vêtements convenables que d'autres trouveront pour Fergus.

Fergus n'a pas l'honneur d'une description stéréotypée de sa beauté. Mais, quoique condamnée comme rebattue par Geoffroi de Vinsauf à la même époque où Guillaume le clerc écrivait ce roman, et déconseillée pour les hommes parce qu'efféminée selon Mathieu de Vendôme, la description de la beauté mâle se retrouve ici et là au cours du XIII ${ }^{\mathrm{e}}$ siècle (voir la n. 13), même assez tardivement. Par exemple, elle apparaît de nouveau chez Floriant (v. 2884-921) dans Floriant et Florete qui, d'ailleurs, subit l'influence des romans de Chrétien ${ }^{50}$. Mais le catalogue descriptif enseigné dans les arts poétiques n'est pas la forme la plus courante de la description des hommes; il n'en devient une variété particulièrement importante que lorsque la beauté physique doit inspirer l'amour, ce qui explique la prédominance de

\footnotetext{
${ }^{46}$ Voir Busby, «Cristal et Clarie: A Novel Romance?» dans Convention and Innovation in Literature, ed. T. D'haen et alii, Amsterdam, 1989, p. 77-103 ; Kelly, op.cit. 1992 [n. 3], p. 294.

${ }^{47}$ L'anomalie a été remarquée; voir Le Roman de Tristan en prose, éd. R. L. Curtius, t. 2, Leiden, 1976, §515:14-17.

${ }^{48}$ Dans Escanor Galentinet s'habille grossièrement, mais c'est pour se déguiser quand il projette la trahison (v. 7866-911).

${ }^{49}$ Cf. Perceval, v. 1012-13 : «se li vallés est niches, / S'est il espoir molt gentix hom ». Voir aussi Chevalier deux épées, v. 1569-75, 1605-09.

${ }^{50}$ Busby, «The Intertexual Coordinates of Floriant et Florete », French Forum, 20, 1995, p. 261-77; Combes et Trachsler, éd., Floriant, p. xxiv-xxvii.
} 
telles descriptions de belles femmes dans les romans arthuriens. Matthieu de Vendôme donne différents exemples de personnes - pape (sans nom propre), César comme empereur, Ulysse comme guerrier, Hélène comme beauté, etc. Il veut que les attributs qu'on leur invente correspondent à leur caractère. Le nom propre doit désigner toute personne de la même condition, comme on dirait «c'est un César, un Ulysse, une belle Hélène $»^{51}$.

Revenons au blandus amator uxoris écarté comme caractéristique du roi et condamné par Jean de Garlande. Ces critères ont-ils été modifiés pour Erec? Sans doute Chrétien pouvait adapter son art aux goûts de ses publics, et ceux-ci appréciaient les chevaliers amants. Donc, avant de tirer quelques conclusions sur les adaptations romanesques de la formule traditionnelle de la description, examinons quelques illustrations du chevalier amoureux dans les romans en vers. Commençons par Gauvain. Si l'Erec le nomme le meilleur chevalier de la Table Ronde, ce n'est pas parce qu'il est un stéréotype consommé. Gauvain aime, mais pas trop, comme dans ses rapports avec Lunete ${ }^{52}$; il défend les femmes, parfois à tort, comme dans le contentieux entre les filles du Sire de la Noire Epine; il participe aux tournois, mais peut s'en abstenir pour observer les exploits des autres, ce qui arrive dans la Charrette (v. 5952-60) et Perceval (v. 5095-107). Il peut être accusé, à tort selon lui, d'avoir tué un seigneur, mais doit se défendre. Si Gauvain excelle, n'est-ce pas parce qu'il est toujours parfaitement courtois ? ${ }^{53}$ S'il y a une anomalie chez lui, c'est qu'il refuse rarement de se nommer, trait qui distingue le neveu d'Arthur des autres chevaliers de la Table Ronde chez qui la coutume est de rester incognito quand ils sont en quête.

Mais dans les romans écrits après Chrétien, on trouve des anomalies semblables dans la description idéale. Dans Rigomer, Lancelot, toujours incognito dans la Charrette, accepte de s'identifier chaque fois qu'on le lui demande; c'est Gauvain qui refuse de le faire, un changement important dans ce trait conventionnel de sa conduite chevaleresque. Dans l'Âtre périlleux et le Chevalier aux deux épées, on croit mort le neveu d'Arthur ${ }^{54}$. Gauvain estime par conséquent avoir perdu son identité ; il ne peut plus se nommer, comme s'il était vraiment mort. La perte du nom l'oblige à faire ce que la grande majorité des chevaliers font dans les romans

\footnotetext{
${ }^{51} \mathrm{Ne}$ nomen proprium preponderet, ceteris personis eiusdem conditionis... intelligatur attributum, ut nomen speciale generalis nominis vicarium ad maneriem rei, non ad rem maneriei reducatur (Ars versificatoria, §1.60). Sur ce genre de « spécialité », voir Kelly, «La Spécialité dans l'invention des topiques», Archéologie du Signe, éd. L. Brind'Amour et E. Vance, Toronto, 1983, p. 101-25. C'est peut-être dans ce sens qu'on doit comprendre cette observation exprimée par Guigemar: "Femmes se resemblent asez» (v. 779, Marie de France, Les Lais, éd. J. Rychner, Paris, 1968).

${ }^{52}$ C'est aussi la portée de son conseil à Yvain (v. 2484-502).

${ }^{53}$ Kelly, "Gauvain and fin'amors in the Poems of Chrétien de Troyes », Studies in Philology, 67, 1970, 453-60 ; Busby, Gauvain in Old French Literature, Amsterdam, 1980, ch. 2-3.

${ }^{54}$ Voir A. Combes, «L'Âtre périlleux : cénotaphe d'un héros retrouvé », Romania, 113, 1992 95, 140-74 ; Rockwell, «Appellation contrôlée : Motif Transfer and the Adoption of Names in the Chevalier as deus espees », "Par la soie amisté »: Essays in Honor of Norris J. Lacy, Amsterdam, Atlanta, 2000, p. 435-52.
} 
arthuriens: se réaliser en recomposant son identité traditionnelle au moyen d'exploits chevaleresques.

Keu est connu comme sénéchal médisant et sarcastique. Sa mauvaise langue fait rire les chevaliers qui s'amusent aux dépens du sénéchal en le taquinant. Comme dans le cas de Gauvain, quelques romans adaptent son type modèle, soit en faisant du médisant un sénéchal profondément méchant et meurtrier dans Yder, soit, dans l'Escanor, en l'anoblissant sous l'influence de la fine amour et du mariage avec Andrivete, fille du roi de Northumberlande ${ }^{55}$. Il fait preuve de prouesse inspirée par l'amour dans le tournoi de Beaubourch ; mais lorsqu'il croit Andrivete infidèle, Keu retombe dans son rôle traditionnel en donnant expression à une violente misogynie ${ }^{56}$.

Chez Chrétien, le chevalier se réalise d'habitude dans les quêtes et les errances; dans les romans du XIII ${ }^{\mathrm{e}}$ siècle, c'est souvent dans les sièges et les tournois qu'il révèle sa supériorité. La révélation ou la découverte du nom est un moment critique dans la réalisation du potentiel chevaleresque. On pense aux cas de Guinglain dans le Bel Inconnu, de Meriadeuc dans Le Chevalier aux deux épées, voire de Lancelot du Lac dans le Chevalier de la charrette et de Perceval dans le Conte du graal. En apprenant son nom, le chevalier acquiert une identité dans le sens où l'entend Mathieu de Vendôme : le nom révèle quelque chose de bien ou de mal chez la personne ${ }^{57}$. Les qualités ou les défauts sont ceux du type représenté, en l'occurrence le chevalier, comme le dit Mathieu dans son propos sur le nom propre.

L'idéal stéréotypé se réalise non seulement dans la description statique, qui ne révèle qu'un potentiel, mais aussi dans le déroulement des intrigues romanesques où ce potentiel doit se manifester de manière exemplaire ${ }^{58}$. On constate parfois cet effort dans les quêtes chevaleresques et autres «errances» caractéristiques des romans arthuriens. Pour terminer, - je ne pourrais ici passer en revue tous les exemples qu'on trouve dans les romans en vers, dont l'originalité est parfois surprenante - regardons rapidement un dernier exemple de l'intégration de descriptions stéréotypées et anormales dans les intrigues romanesques. Il s'agit de Durmart le galois, qui décrit comment le chevalier éponyme réalise son potentiel en changeant et s'améliorant tout en connaissant des états d'âme ${ }^{59}$.

Le jeune Durmart est d'une beauté extraordinaire. Le romancier anonyme consacre une cinquantaine de vers à la première description du jeune homme (v. 98148). Mais au début, la carrière de celui-ci n'est guère brillante. Envoyé chez le sénéchal de son père, roi du Pays de Galles, Durmart tombe amoureux de la jeune

\footnotetext{
${ }^{55}$ Vv. 3781-805, 4265-86, 4296-312. Keu paraît brièvement dans Meliador où il est seulement un bon et jeune sénéchal (v. 25627-29, 25676-812); il ne manifeste pas ses traits conventionnels dans la tradition arthurienne. Voir sur le sénéchal, Ménard, op.cit., p. 455-58; Chênerie, op.cit., p. 99-103.

${ }^{56}$ Une transformation analogue a lieu chez Laudine quand elle croit Yvain inconstant (Kelly, "The Logic of the Imagination in Chrétien de Troyes», The Sower and His Seed: Essays on Chrétien de Troyes, éd. R. T. Pickens, Lexington (KY), 1983, p. 17-20).

${ }^{57}$ Argumentum sive locus a nomine est quando per interpretationem nominis de persona aliquid boni vel mali persuadetur (Ars versificatoria, §1.78). Cf. Bianchini, op.cit., p. 181-86. ${ }^{58}$ Cf. Trachsler, op.cit ; C.-A. Van Coolput-Storms, «Autoportraits de héros, » Conjunctures : Medieval Studies in Honor of Douglas Kelly, Amsterdam, 1994, p. 111.

${ }^{59}$ Cf. Escanor, v. 8246 : « On n'est pas toz jors en un point ».
} 
épouse du vieux sénéchal. Cette liaison n'inspire au romancier que de brèves réflexions sur les mariages mal assortis (cf. v. 165-70). Il insiste plutôt sur la liaison adultère comme cause de recréantise. En effet, son amour courtois ne fait pas de Durmart un Lancelot, ce blandus amator uxoris Arthuri, mais, au contraire, un «beaz malvais» (v. 459): «Dehés ait bealtés sans proëce»(v.458), s'écrie le narrateur! Comme les vêtements d'Enide appauvrie, la conduite de Durmart est mal séante. Par contraste, dans un amour analogue dans Claris et Laris, Claris refuse la recréantise (Claris v. 318) et la trahison que représenterait l'adultère (v. 325-36); il part donc vers la cour d'Arthur sans cesser d'aimer. Chez Claris, il n'y pas d'anomalie semblable à celle de Durmart. Mais ce dernier finit par se reprendre, de manière assez originale d'ailleurs. Un jour il écoute le chant des oiseaux, un acte propre à le faire penser aux plaisirs d'amour dans la poésie amoureuse. Pas chez Durmart (v. 578-92). Rentrant en lui-même, le jeune recréant commence à méditer sur la vie qu'il mène. Cette réflexion lui permet de retrouver la bonne voie. Une nouvelle vie commence. Il quitte son amie, qui rejoint son mari indulgent. Une nouvelle description de Durmart est insérée dans l'intrigue, une description qui accorde l'habillement avec le beau corps (v. 974-92). Le romancier anonyme brosse ce nouveau portrait de Durmart au moment où, armé comme chevalier, il est adoubé. Immédiatement après, il part en quête d'une épouse convenable, non celle d'un autre, mais une dame mariable - la reine d'Irlande.

Pourquoi elle? Fraîchement adoubé, Durmart rencontre un vilain en quittant la cour. Ce vilain, qui rappelle un peu Merlin dans d'autres romans, encourage le nouveau chevalier à chercher la reine d'Irlande parce que la beauté de cette reine est aussi extraordinaire que celle du jeune homme. La beauté de celui-ci est «demesuree», car elle «passe mesure, / Si est mervelle tote pure» (v. 1100-02). Et le vilain de conclure : le plus bel homme doit bien aimer et épouser la plus belle dame. La reine d'Irlande n'est pas encore mariée. En plus, elle est reine, comme Durmart est fils de roi. «Si avenroit bien que vos doi / Eüssiés ensemble ajostees / Vos deus beatés et assemblees» $»(\mathrm{v} .1150-52)$ : une belle conjointure ${ }^{60}$.

Le mariage a lieu, mais après dix mille vers d'errances. Si la beauté demande la prouesse, le beau chevalier doit manifester cette prouesse dans l'action. Durmart entreprend une quête de la reine qui lui permet de surmonter de nombreuses épreuves. Son dernier exploit libère la reine d'Irlande assiégée par un de ses vassaux en rébellion contre sa suzeraine.

Retournons au début de sa quête. Peu après avoir quitté le vilain qui l'a conseillé, Durmart rencontre en fait la reine d'Irlande, mais sans la reconnaître. Il ne se rendra compte de la méprise qu'après avoir été séparé inopinément de celle qu'il cherche. La méprise n'est pas invraisemblable, car la reine ne ressemble guère à une reine quand Durmart la rencontre pour la première fois : elle est seule dans la forêt ${ }^{61}$,

\footnotetext{
${ }^{60}$ Comme Escanor le Beau et son épouse (Escanor, v. 8036-58).

${ }^{61}$ Cf. l'étonnement de Gauvain quand Felinete, la cousine d'Escanor le Beau,arrive après avoir traversé une forêt toute seule (Escanor, v. 20084-89) ; Girard d'Amiens n'évoque pas la coutume de Logres qui protège les femmes qui voyagent seules. Cette coutume, assez importante chez Chrétien, survit dans le roman en prose mais disparaît du roman en vers ; voir D. Maddox, The Arthurian Romances of Chrétien de Troyes: Once and Future Fictions, Cambridge, 1991, p. 36-48, 124-29 ; Chênerie, op.cit., p. 443-46. Plus fréquente dans ces
} 
en route vers un combat pour l'épervier à Landoc, combat qui doit révéler la plus belle. Durmart estime en effet sa beauté. Il trouve que l'inconnue est la plus belle, à l'exception d'elle-même, c'est-à-dire, de la reine d'Irlande qu'il ne reconnaît pas, ne l'ayant jamais vue, pense-t-il.

La plus bele estes, gel sai bien,

De tot cest siecle terrien

Fors la roïne d'Yrelande

De cui veoir sui mout en grande. (vv.1963-66) $)^{62}$

"Vos amés d'estrange maniere» (v. 1984), fait remarquer la reine d'Irlande quand elle apprend que Durmart la cherche. Mais elle s'abstient de s'identifier, préférant attendre jusqu'à ce que Durmart la retrouve dans ses terres. C'est là aussi qu'on apprend le nom, Fenise (v. 14753), de celle qu'on ne connaissait que par le surnom de Reine d'Irlande.

Dans les dix mille vers qui suivent, Durmart poursuit ses errances à la recherche de la belle reine qu'il n'a pas reconnue en la voyant. Les aventures qu'il trouve sur son chemin lui permettent de mettre en œuvre la prouesse complémentaire à sa beauté. Sa prouesse atteint un épanouissement suprême après le mariage. Dans ce dénouement, Durmart défend le pape contre une invasion païenne. Cette prouesse extraordinaire, voire hyperbolique dans le sens de l'Yder, mais digne de sa beauté royale, clôt honorablement la carrière chevaleresque et la belle vie de Durmart le Galois.

Avec ces exemples tirés de quelques romans arthuriens en vers, j'ai cherché à montrer que les romanciers suivent l'exemple de Chrétien de Troyes en adaptant la description stéréotypée au déroulement des aventures qu'ils racontent. Ils tournent donc en récit le stéréotype traditionnel. Pour ce faire, ils introduisent dans leurs descriptions des anomalies que les chevaliers doivent écarter afin d'atteindre la perfection dans le dénouement. Le but paraît avoir été la réalisation intégrale du caractère idéal à travers les aventures achevées dans les errances, les quêtes, les sièges et les intrigues amoureuses: les chevaliers et leurs dames réalisent le stéréotype par les exploits.

Douglas Kelly

University of Wisconsin-Madison

romans est la dame assiégée, souvent avec la menace d'un mariage indésirable ou de viol (Chênerie, op.cit., p. 446-51) ; voir aussi H. Newstead, "The Besieged Ladies in Arthurian Romance », PMLA, 63, 1948, p. 803-30.

${ }^{62}$ On se rappelera encore les paroles de Guigemar au moment où il revoit sa dame, mais doute de ce qu'il voit, car «Femmes se resemblent asez» . 